\title{
Nasal continuous positive airway pressure: influence on digital volume pulse in obstructive sleep apnoea patients
}

\author{
Alexandra Scholze*,\#, Stephanie Lamwers ${ }^{+}$, Martin Tepel ${ }^{*, \#, *}$ and Bernd M. Sanner ${ }^{+}$
}

ABSTRACT: Obstructive sleep apnoea (OSA) is linked to increased cardiovascular risk. This risk can be reduced by nasal continuous positive airway pressure (nCPAP) treatment. As OSA is associated with an increase of several vasoconstrictive factors, we investigated whether nCPAP influences the digital volume pulse wave.

We performed digital photoplethysmography during sleep at night in 94 consecutive patients who underwent polysomnography and 29 patients treated with nCPAP. Digital volume pulse waves were obtained independently of an investigator and were quantified using an algorithm for continuous automated analysis.

In patients with OSA and an apnoea/hypopnoea index (AHI) of $>10$ events $\cdot \mathrm{h}^{-1}$, a significant vasoconstriction was observed during the night $(p<0.0001$ by Friedman's test). A significant positive correlation existed between vasoconstriction and $\mathrm{AHI}$ (Spearman correlation, $r=0.27 ; p<0.01 ; n=94$ ) and the arousal index (Spearman correlation, $r=0.21 ; p<0.05 ; n=94$ ). After 6 months of nCPAP treatment, the AHI was significantly reduced from $27 \pm 3$ events $\cdot h^{-1}$ to $4 \pm 2$ events $\cdot h^{-1}$ (each $\left.n=29 ; p<0.001\right)$ and vasoconstriction during the night was significantly reduced from $10 \pm 3 \%$ to $3 \pm 1 \%(p<0.01)$.

We show changes in the reflective index during the night consistent with vasoconstriction in patients with OSA, which are significantly reduced after 6 months of nCPAP treatment.

KEYWORDS: Digital volume pulse wave, nasal continuous positive airway pressure, obstructive sleep apnoea, vasoconstriction

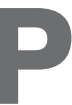

atients with symptomatic obstructive sleep apnoea (OSA) are characterised by repeated episodes of upper-airway occlusion during sleep, with consequent excessive daytime sleepiness and impairment of quality of life $[1,2]$. Patients with untreated OSA have increased cardiovascular morbidity and mortality, whereas treatment with nasal continuous positive airway pressure (nCPAP) reduces cardiovascular risk in these patients $[3,4]$. The pathophysiology of OSA and its adverse effects on the cardiovascular system remain complex. Apnoea and hypopnoea cause temporary elevations in blood pressure in association with hypoxia and sympathetic activation. Intermittent hypoxia causes sympathetic nervous system overactivity, activation of the renin-angiotensin system, oxidative stress and endothelial dysfunction [5-7]. The digital volume pulse provides additional information to peripheral pressure pulse. Its contour is determined mainly by characteristics of the systemic circulation, including pressure wave reflection. Increased pulse wave reflection contributes to disturbances of heart-vessel coupling and finally to the development of cardiovascular disease $[8,9]$.

Treatment of OSA is aimed mainly at preventing the collapse of upper airways, reducing the number of episodes of apnoea and hypopnoea, and reducing the number of oxyhaemoglobin desaturation episodes during sleep. Treatment using $\mathrm{nCPAP}$ reduces the episodes of apnoea and hypopnoea. Several, but not all, investigators have shown that $\mathrm{nCPAP}$ reduces elevated blood pressure [10-12]. Beneficial effects of nCPAP treatment also include reduced activation of sympathetic nervous system [13, 14]. However, there are very limited data on arterial vascular behaviour during nCPAP treatment. We therefore performed noninvasive monitoring of the digital volume pulse wave during the night in OSA with and without nCPAP treatment.

\section{METHODS}

\section{Patients}

We prospectively investigated the digital volume pulse wave in 94 consecutive patients with

\section{AFFILIATIONS}

*0dense University Hospital, Dept of Nephrology,

\#University of Southern Denmark, Institute of Molecular Medicine, Cardiovascular and Renal Research, "Institute for Clinical Research, Odense, Denmark.

${ }^{+}$Medizinische Klinik, Bethesda Krankenhaus, Wuppertal, Germany.

\section{CORRESPONDENCE}

B.M. Sanner

Bethesda Krankenhaus, Medizinische Klinik, Hainstr. 35, 42109 Wuppertal Germany

E-mail. Bernd.Sanner@

Bethesda-Wuppertal.de

Received:

March 252011

Accepted after revision: Aug 182011

First published online:

Sept 012011 
suspected obstructive sleep-related breathing disorders who were admitted to our sleep laboratory through unselected referral from primary or secondary care physicians. Information about sleep history was recorded at baseline and at the time of the final polysomnographic studies. Subjects were studied in a quiet, temperature-controlled room.

The study protocol was approved by the local ethics committee (University of Bochum, Bochum, Germany). Written informed consent was obtained from all patients before entry into the study.

\section{Polysomnography}

94 consecutive patients underwent overnight polysomnography for screening for OSA syndrome according to standardised methods [15-17]. According to the patient's bedtime behaviour, polysomnography and digital photoplethysmography were individually started and finished to allow undisturbed sleep. Baseline measurements were performed with awake patients lying in the supine position before the start of the polysomnography.

An 18-channel polysomnographic recording system (SOHNOlab; Weinmann, Hamburg, Germany) was used to assess sleep state and respiratory and cardiac variables. Continuous recordings included electroencephalography, electro-oculography, electrocardiography, and submental and leg electromyography. The thoracic/abdominal respiratory movements were detected using standard belts with piezoelectric sensors (Weinmann's Somnolab equipment).

Sleep was staged manually using standard methods [15]. Arterial oxyhaemoglobin saturation, oral and nasal airflow, tracheal sounds, and rib-cage and abdominal respiratory motion were used to assess episodes of sleep-disordered breathing. Polysomnography records were inspected visually in 30-s periods for episodes of abnormal sleep, breathing and oxygenation. According to the commonly used clinical criteria, a breathing event during objectively measured sleep was defined as abnormal if either a complete cessation of airflow lasting $\geqslant 10 \mathrm{~s}$ took place (apnoea) or a reduction in respiratory airflow of $\geqslant 50 \%$ of the airflow lasting $>10 \mathrm{~s}$ associated with either an arousal or a desaturation of $>4 \%$ could be discerned (hypopnoea). For the detection of apnoeas/hypopnoeas, respiratory flow nasal cannulas were used.

Obstructive apnoea was defined as the absence of airflow in the presence of paradoxical chest-wall motion. The apnoea/ hypopnoea index (AHI) was defined as the average number of episodes of apnoea and hypopnoea events per hour of objectively measured sleep and was the summary measurement of the occurrence of sleep-disordered breathing. The oxygen desaturation index (ODI) was calculated by dividing the total number of oxygen desaturations by the total sleep time, with desaturation defined as a $\geqslant 10 \mathrm{~s}$ reduction in oxygen saturation $\geqslant 4 \%$ of baseline.

In 29 patients with symptomatic OSA, nCPAP treatment was begun. Patients were advised to retain their usual behaviours, including diet and daily activity. Patients were educated by trained personnel to use their continuous postitive airway pressure (CPAP) device as recommended.
CPAP treatment was performed using devices from Respironics (Murrysville, PA, USA) and Weinmann. Commercially available masks were used and the optimum size was determined individually before treatment. All CPAP titrations were performed in hospital during a second laboratory polysomnography night attended by trained personnel. The majority of patients $(n=23)$ received conventional CPAP with a fixed pressure; four patients received CPAP with expiratory pressure relief technology and two patients received auto-positive airway pressure (PAP). Conventional CPAP titration was performed manually, started at $4 \mathrm{cmH}_{2} \mathrm{O}$ and increased in steps of $1 \mathrm{cmH}_{2} \mathrm{O}$ until removal of apnoeas, hypopnoeas, flow limitations, oxygen desaturations and snoring in all sleep stages and body positions had been acheived. The pressure obtained thereby was considered to be the optimal. Using expiratory relief technology, the expiratory PAP applied can be kept below the CPAP. For auto$\mathrm{PAP}$, the minimal pressure was kept at $5 \mathrm{cmH}_{2} \mathrm{O}$ (pressure range 5-14 $\mathrm{cmH}_{2} \mathrm{O}$ ). The pressure was set to start automatically after $20 \mathrm{~min}$ of adaptation. The devices used monitored snoring, flow and impedance. These parameters are used to detect respiratory events and the pressure is adjusted automatically. The automatic pressure profiles of the devices were considered acceptable if the recording period of the auto-PAP device was $\geqslant 6 \mathrm{~h}$, the mean leak was $<0.4 \mathrm{~L} \cdot \mathrm{s}^{-1}$ and the total sleep time was $\geqslant 5 \mathrm{~h}$. Patients reported proper adherence to CPAP therapy. None of the patients interrupted CPAP therapy during the 6-month interval. Another polysomnographic evaluation was performed 6 months after starting nCPAP treatment.

\section{Digital photoplethysmography}

Digital volume pulse waves were continuously quantified by the reflective index obtained by noninvasive digital photoplethysmography and an algorithm for continuous, investigator-independent, automatic analysis. The reflective index was determined according to SCHOLZE et al. [18] with minor modifications. Digital volume pulse waves were measured by the transmission of red and infrared light through the finger pulp of the third digit using a conventional pulse oximeter with a sampling frequency of $300 \mathrm{~Hz}$, which is then further processed by the internal software at a frequency of $25 \mathrm{~Hz}$. The pulsatile component results in a reduction of transmitted light due to the accompanying increase of optical density and path length of the illuminated tissue. The changes in light intensity are measured by the photodetector and correlate to the digital volume pulse wave. The maximum of each digital volume pulse wave is determined automatically and set to 100 . The reflective index was calculated from the mean of the 5 th to the 10th data point after the maximum of the digital volume pulse wave. This area covers the diastolic shoulder region to the dicrotic wave, which is in part formed by pulse waves reflected back from peripheral vascular sites of impedance mismatch. As indicated in our previous studies, short-term changes of the reflective index reflect vasoconstriction or vasodilatation [18, 19]. Earlier studies from our group indicated that the reflective index shows a significant positive correlation with invasively determined systemic vascular resistance by pulmonary artery catheter (Spearman correlation, $\mathrm{r}=0.64, \mathrm{p}<0.01, \mathrm{n}=20$ ). In mechanically ventilated ICU patients under anaesthesia with midazolam and fentanyl, there was a significant correlation between lower $\mathrm{pH}$ in the arterial blood gas analysis and vasodilation indicated by a lower reflective index (Spearman correlation, $\mathrm{r}=0.22, \mathrm{p}<0.05 ; \mathrm{n}=102$ ) but not with arterial oxygen tension or arterial carbon dioxide tension. 

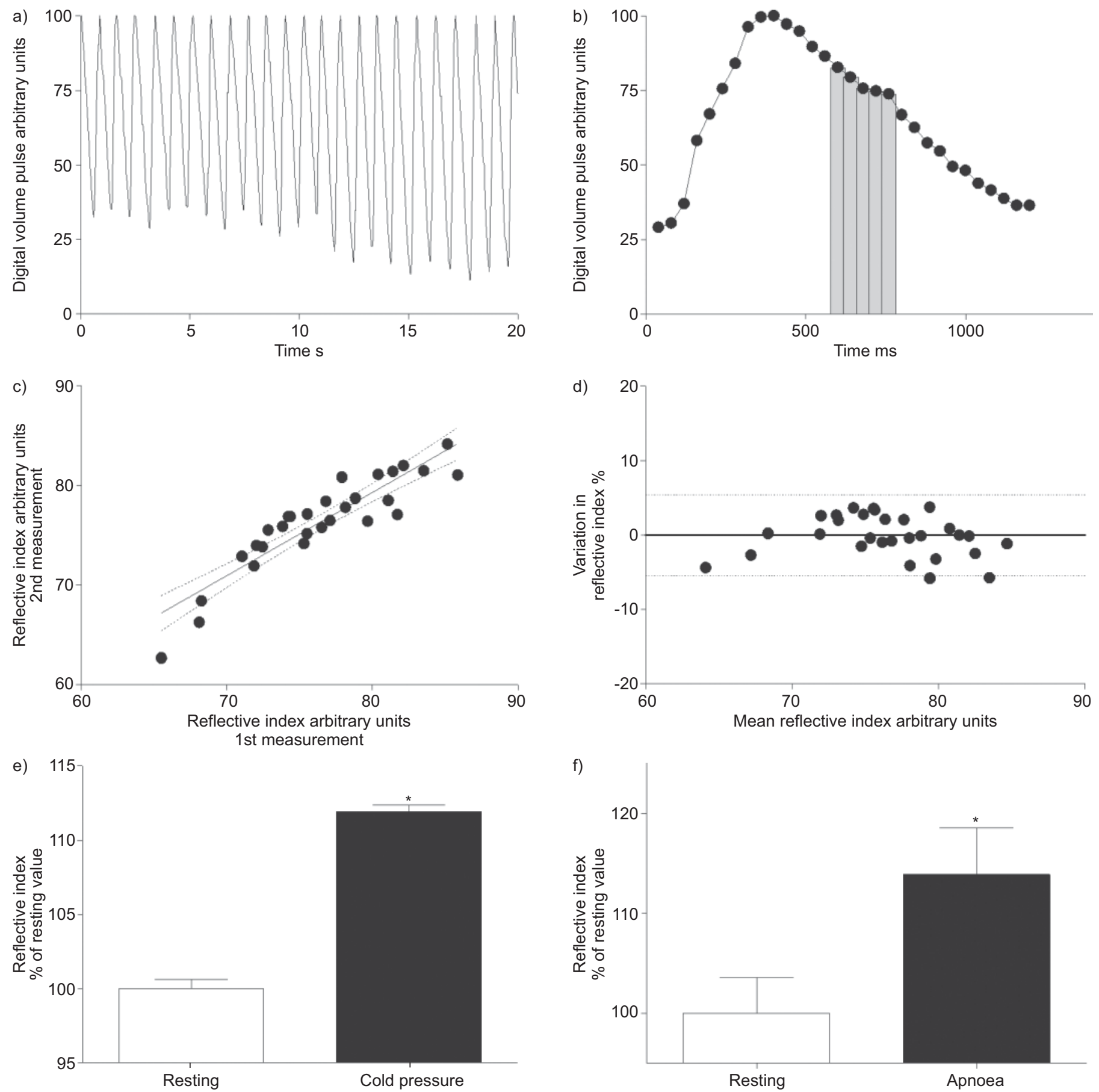

FIGURE 1. Determination of the reflective index by fingertip photoplethysmography. a) Typical digital volume pulse waves obtained by photoplethysmography during $20 \mathrm{~s}$ are shown. Pulse waves were measured by the transmission of red and infrared light through the finger pulp. b) One typical pulse wave is depicted. The maximum of the digital pulse wave was determined. The reflective index was calculated from the mean of the 5 th to the 10th data point after the maximum of the digital pulse wave (shaded area) covering the typical "shoulder region" of the diastolic pulse wave decay. c) Regression curve for first and second measurements of the reflective index. Spearman correlation, $r=0.92 ; p<0.001 ; n=30$. d) Bland-Altman plot quantifying the average discrepancy between two measurements; the bias and $95 \%$ limit of agreement are indicated by lines. e) Bar graph showing summary data of the reflective index under resting conditions and during cold-pressure test in healthy subjects. Data are presented as mean $\pm \operatorname{SEM}(n=3)$. f) Bar graph showing summary data of the reflective index under resting conditions and during apnoea in healthy subjects. *: $p<0.05$ compared to resting conditions.

Data of the reflective index obtained from all digital volume pulse waves were averaged every $2.5 \mathrm{~min}$. The reflective index values shown in figures 2 and 4 and used for the statistical analyses were obtained at every full hour between midnight and $05.00 \mathrm{~h}$ in all patients and, therefore, give the respective reflective indices at a given time of day. 


\begin{tabular}{|c|c|c|c|c|}
\hline \multirow{2}{*}{ TABLE 1} & \multicolumn{4}{|c|}{$\begin{array}{l}\text { Clinical and biochemical characteristics of } 94 \\
\text { patients that underwent polysomnography }\end{array}$} \\
\hline & & $A H I<10$ & $A H I>10$ & $\mathrm{p}$-value \\
\hline \multicolumn{2}{|l|}{ Subjects n } & 29 & 65 & \\
\hline \multicolumn{2}{|l|}{ Age yrs } & $57 \pm 2$ & $60 \pm 2$ & 0.26 \\
\hline \multicolumn{2}{|l|}{ Male } & $20(69)$ & $50(77)$ & 0.45 \\
\hline \multicolumn{2}{|c|}{ Body mass index $\mathrm{kg} \cdot \mathrm{m}^{-2}$} & $27.8 \pm 1.0$ & $29.1 \pm 0.6$ & 0.31 \\
\hline \multicolumn{5}{|c|}{ Comorbidities } \\
\hline \multicolumn{2}{|l|}{ Diabetes } & $4(14)$ & $9(14)$ & 1.00 \\
\hline \multicolumn{2}{|c|}{ Hypertension } & $14(48)$ & $34(52)$ & 0.82 \\
\hline \multicolumn{2}{|l|}{ CVD } & $10(35)$ & $18(28)$ & 0.63 \\
\hline \multicolumn{2}{|l|}{ Smoking } & $12(41)$ & $18(28)$ & 0.23 \\
\hline \multicolumn{2}{|c|}{ Systolic blood pressure $\mathrm{mmHg}$} & $132 \pm 5$ & $136 \pm 3$ & 0.27 \\
\hline \multicolumn{2}{|c|}{ Diastolic blood pressure $\mathrm{mmHg}$} & $84 \pm 3$ & $84 \pm 2$ & 0.67 \\
\hline \multicolumn{2}{|c|}{ Pulse pressure $\mathrm{mmHg}$} & $48 \pm 4$ & $53 \pm 2$ & 0.21 \\
\hline \multicolumn{2}{|c|}{ Leukocytes cells $\cdot \mathrm{nL}^{-1}$} & $7.9 \pm 0.4$ & $7.6 \pm 0.3$ & 0.63 \\
\hline \multicolumn{2}{|c|}{ Haemoglobin $\mathbf{g} \cdot \mathbf{d L}^{-1}$} & $14.8 \pm 0.3$ & $14.5 \pm 0.2$ & 0.51 \\
\hline \multicolumn{2}{|c|}{ Platelets cells $\cdot \mathrm{nL}^{-1}$} & $263 \pm 10$ & $259 \pm 9$ & 0.53 \\
\hline \multicolumn{2}{|c|}{ Serum creatinine $\mathrm{mg} \cdot \mathrm{dL}^{-1}$} & $1.0 \pm 0.0$ & $1.0 \pm 0.0$ & 0.45 \\
\hline \multicolumn{2}{|c|}{ Serum sodium $\mathrm{mmol} \cdot \mathrm{L}^{-1}$} & $140 \pm 1$ & $140 \pm 0$ & 0.83 \\
\hline \multicolumn{2}{|c|}{ Serum potassium $\mathrm{mmol} \cdot \mathrm{L}^{-1}$} & $4.2 \pm 0.1$ & $4.1 \pm 0.1$ & 0.43 \\
\hline \multicolumn{2}{|c|}{ Serum calcium $\mathrm{mmol} \cdot \mathrm{L}^{-1}$} & $2.40 \pm 0.03$ & $2.35 \pm 0.01$ & 0.07 \\
\hline \multicolumn{2}{|c|}{ Glucose $\mathrm{mmol} \cdot \mathrm{L}^{-1}$} & $6.9 \pm 0.6$ & $7.6 \pm 0.4$ & 0.15 \\
\hline \multicolumn{2}{|c|}{ Total cholesterol $\mathrm{mq} \cdot \mathrm{dL}^{-1}$} & $212 \pm 9$ & $215 \pm 6$ & 0.94 \\
\hline \multicolumn{2}{|c|}{ Triglycerides $\mathrm{mg} \cdot \mathrm{dL}^{-1}$} & $152 \pm 20$ & $211 \pm 20$ & 0.09 \\
\hline \multicolumn{2}{|c|}{ C-reactive protein $\mathrm{mg} \cdot \mathrm{dL}^{-1}$} & $0.6 \pm 0.3$ & $0.4 \pm 0.1$ & 0.19 \\
\hline \multicolumn{5}{|l|}{ Medication } \\
\hline \multicolumn{2}{|l|}{ ACE/AT } & $11(38)$ & $24(37)$ & 1.00 \\
\hline \multicolumn{2}{|l|}{$\beta$-blockers } & $8(28)$ & $19(29)$ & 1.00 \\
\hline \multicolumn{2}{|c|}{ Calcium channel inhibitors } & $3(10)$ & $10(15)$ & 0.75 \\
\hline \multicolumn{2}{|l|}{ Diuretics } & $8(28)$ & $16(25)$ & 0.80 \\
\hline \multicolumn{2}{|c|}{ Antiplatelet drugs } & $7(24)$ & $20(31)$ & 0.62 \\
\hline
\end{tabular}

Data are presented as mean \pm SEM or $n(\%)$, unless otherwise stated. CVD: presence of or a history of coronary artery disease, stroke or peripheral arterial vascular disease; ACE/AT: angiotensin-converting enzyme inhibitors/angiotensin receptor antagonists. Patients were grouped according to their respective apnoea/hypopnoea index (AHI).

\section{Statistics}

Descriptive statistics for continuous variables are given as mean \pm SEM. Differences between groups were analysed by nonparametric Mann-Whitney test. Relationships between parameters were assessed using nonparametric partial Spearman correlations. Reproducibility data are given as BlandAltman plots that depict percentage differences between two measurements plotted against the mean of two measurements. Bias and 95\% limit of agreement are given. Changes of the reflective index during the night were analysed by nonparametric Friedman's test with Dunn's multiple comparison post hoc test. Analyses were performed with GraphPad prism software (version 5.0, GraphPad Software, San Diego, CA, USA). All statistical tests were two-sided. A two-sided p-value of $<0.05$ was considered to indicate statistical significance.

\section{RESULTS}

Digital volume pulse waves and the diastolic shoulder region used for the determination of the reflective index are depicted in figures $1 \mathrm{a}$ and $\mathrm{b}$. To demonstrate the reliability of reflective index measurements repeated determinations were performed (figs $1 \mathrm{c}$ and $\mathrm{d}$ ). There was a good agreement between the first and the second determination of the reflective index $(n=30 ; r=0.92$; $\mathrm{p}<0.001)$. Analysing the data according to Bland-Altman confirmed a good reproducibility of measurements (bias, $-0.1 \%$; $95 \%$ limit of agreement, $-5.5-5.4 \%)$. To illustrate how vasoconstriction results in an increased reflective index, digital volume pulse waves were measured using photoplethysmography during systemic vasoconstriction induced by cold-pressure tests in healthy subjects. As shown in figure 1e, the reflective index significantly increased during cold-pressure tests from $100 \pm 1 \%$ to $112 \pm 1 \%$ arbitrary units $(n=3 ; p<0.05)$ indicating vasoconstriction. In healthy subjects, voluntary apnoea significantly increased the reflective index from $100 \pm 4 \%$ to $114 \pm 5 \%$ arbitrary units $(\mathrm{p}<0.05)$, consistent with vasoconstriction during apnoea. During apnoea there was also an increase of muscle sympathetic nerve activity (18) from 9 to 28 bursts $\cdot \mathrm{min}^{-1}$.

The clinical, biochemical and polysomnographic characteristics of 94 patients that underwent polysomnography for screening of OSA are shown in table 1 . The changes of the reflective index during the night for all 94 patients are summarised in figure 2a. The baseline reflective index $(100 \%)$ was obtained when patients were awake lying in the supine position at the start of the polysomnography. In patients with an $\mathrm{AHI}<10$ events $\cdot \mathrm{h}^{-1}$, no significant change of the reflective index was observed during the night ( $n=29 ; p>0.05$; fig. $2 b)$. After the exclusion of females, the results remained unchanged $(n=20 ; p>0.05)$. In contrast, 65 patients had OSA with an AHI $>10$ events $\cdot h^{-1}$. In these patients, the reflective index significantly increased to $109 \pm 2 \%$ during the night $(n=65 ; p<0.0001$; fig. $2 b)$. In the subgroup of males with AHI $>10$ events $\cdot h^{-1}$, the reflective index significantly increased to $109 \pm 2 \% \quad(n=50 ; p<0.0001)$. We observed a significant correlation between the AHI and the change of the reflective index during the night $(\mathrm{r}=0.27 ; \mathrm{p}=0.01 ; \mathrm{n}=94)$. After the exclusion of females, this correlation remained significant $(\mathrm{r}=0.29 ; \mathrm{p}=0.01 ; \mathrm{n}=70)$. The arousal index was also significantly associated with the change of the reflective index during the night $(\mathrm{r}=0.21, \mathrm{p}=0.03, \mathrm{n}=94$; and $\mathrm{r}=0.27, \mathrm{p}=0.02, \mathrm{n}=70$ for males only). With increasing AHI or arousal index, an increased reflective index was observed, consistent with an increased vasoconstriction in more severe OSA.

From a cardiovascular point of view, oxygen desaturation events $>4 \%$ during sleep are of special interest; therefore, ODI $>4 \%$ was also analysed. There was a significant correlation between the ODI and the change of the reflective index during the night $(\mathrm{r}=0.22 ; \mathrm{p}=0.02 ; \mathrm{n}=119)$. The same correlation was found in the subgroup of male patients $(r=0.25 ; \mathrm{p}=0.02 ; \mathrm{n}=87)$.

To obtain information about the influence of sleep stage on the reflective index, we analysed the reflective index in relation to sleep stage at $01.00 \mathrm{~h}$. Sleep stages were determined visually according to ReChTSCHAFFEN and KALES [20]. Delta sleep comprises sleep stages 3 and 4 . We found a significantly higher reflective index, consistent with higher vasoconstriction, in sleep stage 1 compared with rapid eye movement (REM) sleep ( $n=94 ; \mathrm{p}<0.05$; fig. 3 ).

Of the 94 patients that underwent polysomnography, 13 patients had an $\mathrm{AHI}<5,19$ patients were found to have an $\mathrm{AHI}>5$ but 

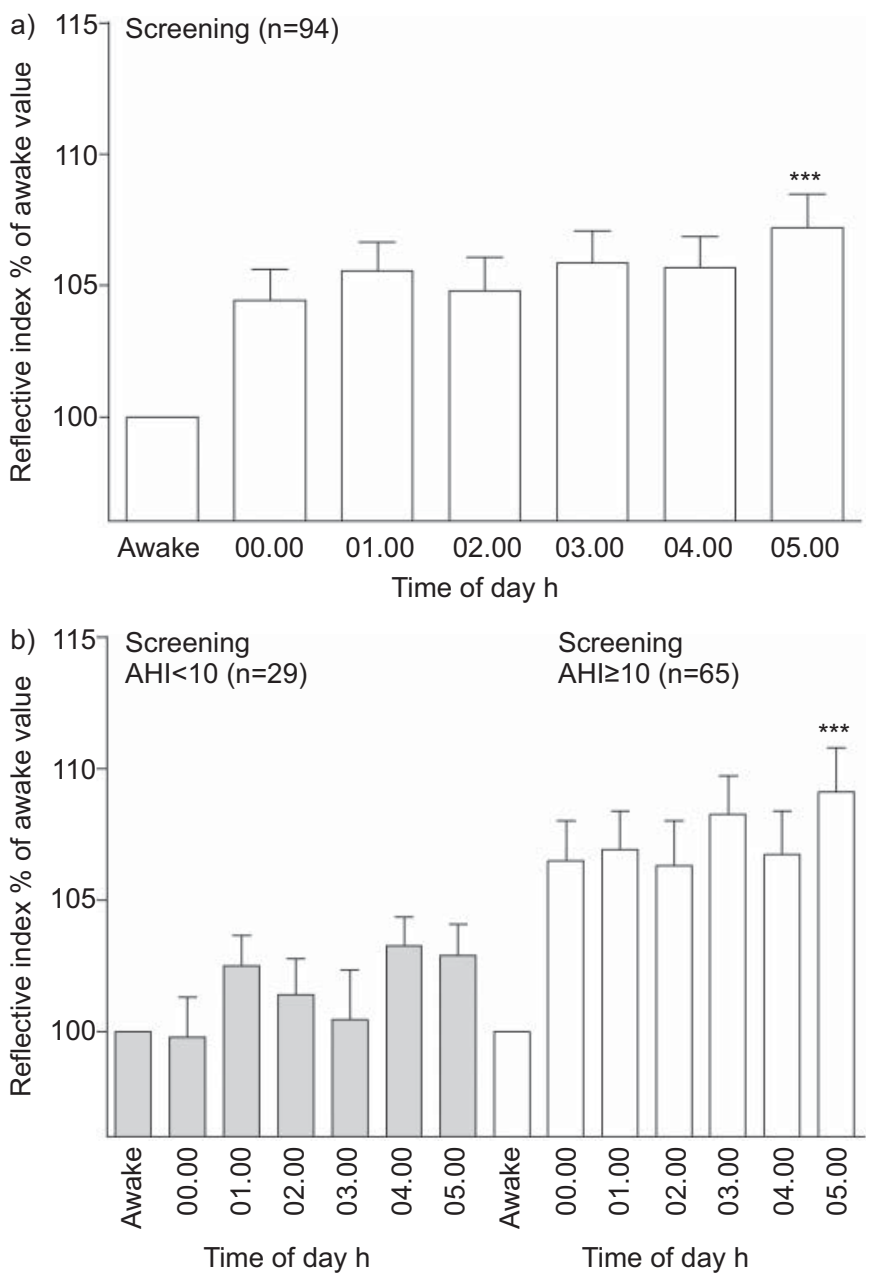

FIGURE 2. Reflective index in patients that underwent polysomnography. a) Bar graph showing the reflective index in all 94 patients that underwent polysomnography for screening of obstructive sleep apnoea. ${ }^{* \star *}: p<0.001$ by Friedman test with Dunn's multiple comparison post hoc test compared with patients awake in the lying position before the start of the polysomnography. b) Comparison of the reflective index in 29 patients with an apnoea/hypopnoea index (AHI) of $<10$ events $\cdot h^{-1}$ and 65 patients with an $\mathrm{AHI}$ of $>10$ events $\cdot h^{-1}$ during the night. ${ }^{* *}: p<0.0001$ by Friedman test with Dunn's multiple comparison post hoc test compared with patients awake in the lying position before the start of the polysomnography.

were asymptomatic for OSA, and 62 patients were symptomatic and in need of treatment. Asymptomatic patients reported no significant daytime sleepiness (Epworth Sleepiness Scale (ESS) $<10$ ), insomnia, subjective sleep disturbances, snoring or other OSA-associated symptoms.

Of the 62 patients for whom nCPAP treatment was recommended after the polysomnography screening, only 29 patients were available to follow-up due to administrative restrictions. Those patients and asymptomatic patients were compared and are characterised in table 2.

In 29 patients with symptomatic OSA, nCPAP treatment was started and polysomnography was repeated after 6 months. CPAP adherence was measured as number of CPAP hours per night recorded as machine run time from the time counter

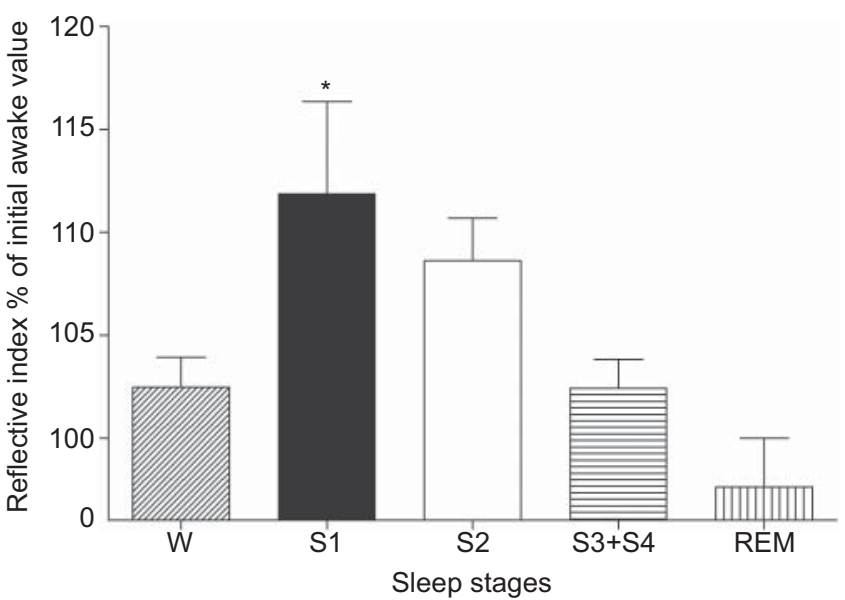

FIGURE 3. Reflective index values in relation to patients' sleeping stage at 01.00 h. Bar graph showing summary data for reflective indices at sleep stages $W$ (awake), S1, S2, S3+S4 (delta sleep) and rapid eye movement (REM) in 94 patients that underwent polysomnography screening. Data are given as a percentage of the reflective index of patients awake in the lying position before the start of the polysomnography. *: $p<0.05$ by Kruskal-Wallis test with Dunn's multiple comparison post hoc test. Sleep stage 1 showed a significantly higher reflective index compared with REM sleep, consistent with a higher vasoconstriction in sleep stage 1 .

internal to the CPAP device. Mean CPAP adherence was $5.6 \pm 0.4 \mathrm{~h} \cdot$ night $^{-1}$. The clinical, biochemical and polysomnographic characteristics of CPAP treated patients are shown in table 3. After nCPAP treatment, the AHI was significantly reduced from $24 \pm 4$ to $4 \pm 2$ events $\cdot h^{-1}(p<0.001)$. In the subgroup of male patients, the AHI was significantly reduced from $26 \pm 4$ to $5 \pm 3$ events $\cdot h^{-1}(n=20, p<0.001)$ after nCPAP treatment. The ODI $>4 \%$ was reduced by nCPAP from $26 \pm 3$ in all treated patients and $27 \pm 4$ in male patients to $0 \pm 0$, respectively $(\mathrm{p}<0.0001)$.

Before nCPAP treatment in 29 patients with symptomatic OSA, the reflective index significantly increased during the night to $110 \pm 3 \%$ at $05.00 \mathrm{~h}$ in the morning, indicating vasoconstriction $(n=29 ; p<0.001$; fig. $4 a)$. In the subgroup of male patients with symptomatic OSA, the reflective index significantly increased to $109 \pm 2 \%(n=20 ; p<0.01)$.

After 6 months of nCPAP treatment, the significant increase of the reflective index during the night could no longer be observed $(n=29 ; p>0.05$; fig. $4 b)$. The same result was obtained in the subgroup of male patients $(n=20 ; p>0.05)$. These data are in line with the notion that the successful therapy of OSA by nCPAP was accompanied by a reduction of the reflective index consistent with reduced vasoconstriction during the night.

\section{DISCUSSION}

The present study shows that patients with symptomatic OSA exhibited a significantly increased reflective index consistent with vasoconstriction during sleep at night. The severity of OSA as quantified by the AHI was directly associated with the increase in vasoconstriction. The successful treatment of OSA using nCPAP significantly reduced vasoconstriction, providing one possible explanation for beneficial effects of nCPAP therapy on cardiovascular morbidity in patients with OSA. 
TABLE 2 Characteristics of 19 asymptomatic and 29 symptomatic patients with obstructive sleep apnoea (OSA)

\begin{tabular}{|c|c|c|c|}
\hline Subjects $\mathrm{n}$ & 19 & 29 & \\
\hline Arousal index ${ }^{\#}$ & $11 \pm 1$ & $14 \pm 2$ & 0.22 \\
\hline Sleep efficiency \% & $76 \pm 3$ & $76 \pm 2$ & 0.96 \\
\hline Stage wake min & $102 \pm 16$ & $103 \pm 9$ & 0.59 \\
\hline Sleep stage $2 \%$ of total sleep time & $53.5 \pm 3.1$ & $57.6 \pm 3.2$ & 0.25 \\
\hline Delta sleep stage $3+4 \%$ of total sleep time & $24.0 \pm 2.7$ & $19.9 \pm 2.1$ & 0.27 \\
\hline Sleep stage REM \% of total sleep time & $12.8 \pm 1.5$ & $11.6 \pm 1.4$ & 0.57 \\
\hline Minimum nocturnal $\mathrm{Sa}, \mathrm{O}_{2} \%$ & $83 \pm 1$ & $77 \pm 2$ & $<0.05$ \\
\hline Mean nocturnal $\mathrm{Sa}, \mathrm{O}_{2} \%$ & $94 \pm 0$ & $93 \pm 0$ & $<0.05$ \\
\hline
\end{tabular}

Data are presented as mean $\pm \mathrm{SEM}$, unless otherwise stated. AHI: apnoea/hypopnoea index (mean number of episodes of apnoea and hypopnoea per hour of sleep); REM: rapid eye movement; $\mathrm{Sa}_{1} \mathrm{O}_{2}$ : arterial oxygen saturation; ESS: Epworth Sleepiness Scale; ODI: oxygen desaturation index. ${ }^{*}$ : the arousal index is the mean number of arousal reactions per hour of sleep; " : reflective index at $05.00 \mathrm{~h}$ is given as percentage of the reflective index of the patient awake in the lying position before the start of the polysomnography.

In previous investigations, it was shown that an ODI $\geqslant 4 \%$ was associated with prevalent cardiovascular disease in a large community sample of middle-aged and older subjects [21]. In our study population with a high prevalence of sleepdisordered breathing, we found a significant correlation between the ODI $>4 \%$ and the change of the reflective index during the night. Higher oxygen desaturation indices were associated with a more pronounced increase of the reflective index consistent with more pronounced vasoconstriction during the night. These findings suggest that changes of the reflective index during the night may add information for the assessment of cardiovascular risk in these patients.

TABLE 3 Characteristics of 29 patients with obstructive sleep apnoea before and after nasal continuous positive airway pressure (nCPAP) treatment

\begin{tabular}{|c|c|c|c|}
\hline Characteristic & Before nCPAP & After nCPAP & p-value \\
\hline Subjects $n$ & 29 & 29 & \\
\hline Arousal index ${ }^{\#}$ & $14 \pm 2$ & $10 \pm 1$ & $<0.05$ \\
\hline Sleep efficiency \% & $76 \pm 2$ & $81 \pm 4$ & $<0.05$ \\
\hline Stage wake $\mathrm{min}$ & $103 \pm 9$ & $66 \pm 9$ & $<0.01$ \\
\hline Sleep stage $2 \%$ of total sleep time & $57.6 \pm 3.2$ & $52.6 \pm 3.1$ & 0.35 \\
\hline Delta sleep stage $3+4, \%$ of total sleep time & $19.9 \pm 2.1$ & $27.6 \pm 2.8$ & $<0.05$ \\
\hline Sleep stage REM \% of total sleep time & $11.6 \pm 1.4$ & $14.2 \pm 1.4$ & 0.17 \\
\hline Minimum nocturnal $\mathrm{Sa}, \mathrm{O}_{2} \%$ & $77 \pm 2$ & $86 \pm 1$ & $<0.0001$ \\
\hline Mean nocturnal $\mathrm{Sa}, \mathrm{O}_{2} \%$ & $93 \pm 0$ & $94 \pm 0$ & $<0.0001$ \\
\hline $\mathrm{Sa}, \mathrm{O}_{2}<90 \% \%$ & $19 \pm 4$ & $4 \pm 1$ & $<0.0001$ \\
\hline
\end{tabular}

Data are presented as mean \pm SEM, unless otherwise stated. AHI: apnoea/hypopnoea index (mean number of episodes of apnoea and hypopnoea per hour of sleep); REM: rapid eye movement; $\mathrm{Sa}, \mathrm{O}_{2}$ : arterial oxygen saturation; ODI: oxygen desaturation index. ${ }^{*}$ : the arousal index is the mean number of arousal reactions per hour of sleep; ${ }^{\bullet}$ : reflective index at $05.00 \mathrm{~h}$ is given as percentage of the reflective index of the patient awake in the lying position before the start of the polysomnography. 

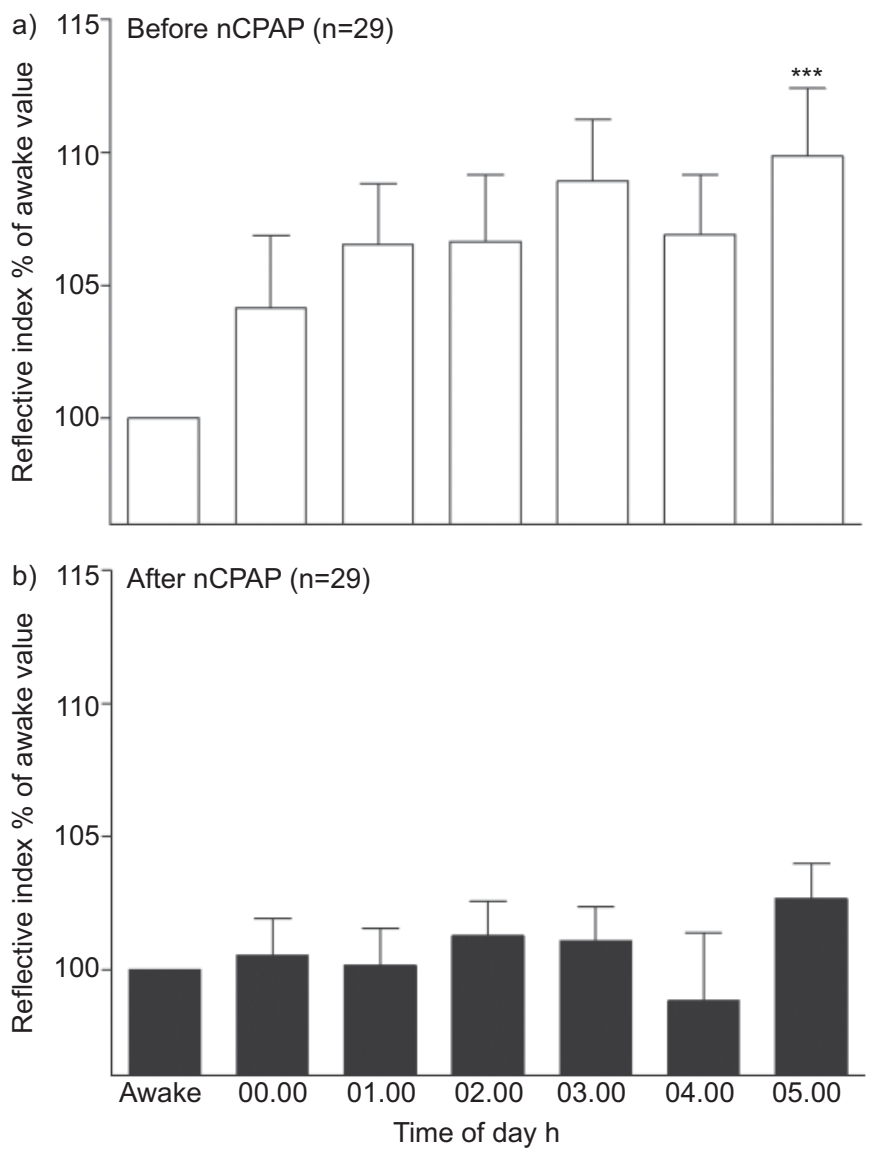

FIGURE 4. Reflective index in 29 patients with obstructive sleep apnoea before and 6 months after treatment with nasal continuous positive airway pressure (nCPAP). a) Bar graph showing the reflective index during the night before nCPAP treatment. ${ }^{* *}: p<0.001$ by Friedman test with Dunn's multiple comparison post hoc test compared with patients awake in the lying position before the start of the polysomnography. b) Bar graph showing the reflective index during the night after nCPAP treatment.

Several mechanisms have been described in patients with OSA that can result in increased vasoconstriction, finally leading to progression of cardiovascular disease. First, increased sympathetic activation induced by nocturnal hypoxia has been reported in normal volunteers and in patients with OSA [22, 23]. In these studies, sympathetic activity was derived from measurements of muscle sympathetic nerve activity. Previous experimental studies from our group already indicated that an elevated sympathetic nerve activity measured by microneurography from the peroneal nerve results in an increased reflective index [18]. In the present study, we confirmed that apnoea increased the reflective index in a similar way to vasoconstriction induced by the cold-pressure test. Therefore, our present results of increased vasoconstriction are in line with previous findings of increased sympathetic activity in patients with OSA. Secondly, patients with OSA show endothelial dysfunction, which predisposes to reduced vasodilatation and increased vasoconstriction. Studies showed that patients with OSA had lower endothelium-dependent flow-mediated dilation compared with control subjects $[24,25]$. Thirdly, patients with OSA had significantly higher plasma levels of potent vasoconstrictors, including endothelin-1 and angiotensin II, compared with healthy controls. Furthermore, the endothelin-1 level was significantly correlated with the AHI [26, 27]. Fourthly, patients with OSA had significantly higher markers of oxidative stress and inflammation, eventually leading to hypertension [28]. Increased reactive oxygen species had also been reported in patients with OSA [29]. Increased serum concentrations of high sensitivity C-reactive protein, interleukin-6, tumour necrosis factor- $\alpha$ and malondialdehyde could be reduced during successful nCPAP treatment [30].

We found a significant influence of sleep stage on the reflective index, with lower reflective index values consistent with less vasoconstriction in deep (delta) sleep and significantly lower reflective index in REM sleep compared with sleep stage 1 . This might be explained by an increase of vagal tone in deep sleep compared with stage 1 sleep and in components of REM sleep [31, 32]. A significant decrease of blood catecholamine concentration (epinephrine and norepinephrine) during REM sleep, despite the well-known increase of muscle sympathetic activity during this stage, has been convincingly demonstrated and discussed by DODT et al. [33]. According to recent guidelines, nCPAP devices are recommended to treat patients with symptomatic OSA to reduce their cardiovascular risk $[2,34]$. The mean CPAP adherence in our study is either in the same range or above the values reached in comparable investigations [35-37]. According to recent literature, mean nCPAP duration of 5-6 h predicts normalisation of the Functional Outcomes of Sleep Questionnaire in $68 \%$ of patients [35]. CPAP use of $5.6 \mathrm{~h}$ per night constitutes the threshold for positive effects on blood pressure in nonsleepy hypertensive patients reported by BARBÉ et al. [36] and was shown to reduce blood pressure and nocturnal sympathetic activity [37].

The present study shows that successful treatment of OSA using nCPAP significantly reduces vasoconstriction during the night. Treatment with nCPAP significantly reduced sympathetic nerve activity and norepinephrine plasma levels in patients with OSA $[14,38]$. The reduction of sympathetic nerve activity could contribute to the reduced vasoconstriction observed in the present study. Furthermore, previous studies showed that effective treatment using nCPAP significantly reduced leptin in patients with OSA [39]. As leptin is able to stimulate sympathetic mechanisms that produce vasoconstriction, the reduced leptin concentrations during nCPAP treatment may ameliorate vasoconstriction [40]. Decreased sympathetic nerve activity and decreased concentrations of vasoconstrictive mediators are probably the underlying mechanisms that finally result in the reduced vasoconstriction we observed 6 months after starting the nCPAP therapy; however, our study did not investigate the underlying mechanisms or mediators. The reduction of nocturnal vasoconstriction causes shifting of the pulse wave reflecting sites to more peripheral areas. As outlined by SAFAR et al. [9], shifting of the pulse wave reflecting sites to more peripheral areas improves heart-vessel coupling and eventually results in a reduced cardiovascular risk. We used an analytical method that allows noninvasive, investigator-independent, automatic analysis enabling monitoring of digital volume pulse during the night. Further studies should be performed to investigate if the observed vascular effects under nCPAP treatment can be used to predict cardiovascular risk reduction in individual nCPAP-treated patients. Mean delta sleep (sleep stages $3+4$ ) in our cohort was $>20 \%$ in asymptomatic OSA, whereas it was lower in patients 
with symptomatic OSA. Delta sleep significantly increased with nCPAP treatment. These results are in agreement with improved delta sleep with CPAP therapy [41, 42]; however, we cannot exclude an overestimation in our reports of delta sleep. Interscorer reliability has been shown to range from 65 to $78 \%$ when applying the criteria of RECHTSCHAFFEN and KALES [20] and NORMAN et al. [43]. All our sleep stage data were evaluated by one scorer.

\section{Conclusion}

In summary, using digital photoplethysmography we have shown a change in the reflective index consistent with increased vasoconstriction during the night in patients with OSA and an AHI $>10$. We show that a successful therapy of symptomatic OSA by nCPAP for 6 months is accompanied by a change in the reflective index consistent with reduced vasoconstriction during the night.

\section{STATEMENT OF INTEREST}

A statement of interest for B.M. Sanner can be found at www.erj. ersjournals.com/site/misc/statements.xhtml

\section{REFERENCES}

1 Pack AI. Advances in sleep-disordered breathing. Am J Respir Crit Care Med 2006; 173: 7-15.

2 Basner RC. Continuous positive airway pressure for obstructive sleep apnea. N Engl J Med 2007; 356: 1751-1758.

3 Marin JM, Carrizo SJ, Vicente E, et al. Long-term cardiovascular outcomes in men with obstructive sleep apnoea-hypopnoea with or without treatment with continuous positive airway pressure: an observational study. Lancet 2005; 365: 1046-1053.

4 Buchner NJ, Sanner BM, Borgel J, et al. Continuous positive airway pressure treatment of mild to moderate obstructive sleep apnea reduces cardiovascular risk. Am J Respir Crit Care Med 2007; 176: 1274-1280.

5 Fletcher EC. Invited review: Physiological consequences of intermittent hypoxia: systemic blood pressure. J Appl Physiol 2001; 90: 1600-1605.

6 Fletcher EC, Orolinova N, Bader M. Blood pressure response to chronic episodic hypoxia: the renin-angiotensin system. J Appl Physiol 2002; 92: 627-633.

7 Lavie L. Obstructive sleep apnoea syndrome-an oxidative stress disorder. Sleep Med Rev 2003; 7: 35-51.

8 Millasseau SC, Kelly RP, Ritter JM, et al. The vascular impact of aging and vasoactive drugs: comparison of two digital volume pulse measurements. Am J Hypertens 2003; 16: 467-472.

9 Safar ME, Levy BI, Struijker-Boudier H. Current perspectives on arterial stiffness and pulse pressure in hypertension and cardiovascular diseases. Circulation 2003; 107: 2864-2869.

10 Sanner BM, Tepel M, Markmann A, et al. Effect of continuous positive airway pressure therapy on 24-hour blood pressure in patients with obstructive sleep apnea syndrome. Am J Hypertens 2002; 15: 251-257.

11 Barbé F, Duran-Cantolla J, Capote F, et al. Long-term effect of continuous positive airway pressure in hypertensive patients with sleep apnea. Am J Respir Crit Care Med 2010; 181: 718-726.

12 Campos-Rodriguez F, Grilo-Reina A, Perez-Ronchel J, et al. Effect of continuous positive airway pressure on ambulatory BP in patients with sleep apnea and hypertension. Chest 2006; 129: 1459-1467.

13 Imadojemu VA, Mawji Z, Kunselman A, et al. Sympathetic chemoreflex responses in obstructive sleep apnea and effects of continuous positive airway pressure therapy. Chest 2007; 131: 1406-1412.
14 Heitmann J, Ehlenz K, Penzel T, et al. Sympathetic activity is reduced by nCPAP in hypertensive obstructive sleep apnoea patients. Eur Respir J 2004; 23: 255-262.

15 American Academy of Sleep Medicine. The AASM Manual for the Scoring of Sleep and Associated events. Westchester, American Academy of Sleep Medicine, 2007.

16 American Thoracic Society, Medical Section of the American Lung Association. Indications and standards for cardiopulmonary sleep studies. Am Rev Respir Dis 1989; 139: 559-568.

17 American Academy of Sleep Medicine Task Force. Sleep-related breathing disorders in adults: recommendations for syndrome definition and measurement techniques in clinical research. Sleep 1999; 22: 667-689.

18 Scholze A, Burkert A, Mardanzai K, et al. Increased arterial vascular tone during the night in patients with essential hypertension. J Hum Hypertens 2007; 21: 60-67.

19 Wittrock M, Scholze A, Compton F, et al. Noninvasive pulse wave analysis for the determination of central artery stiffness. Microvasc Res 2009; 77: 109-112.

20 Rechtschaffen A, Kales A, eds. A Manual of Standardized Terminology, Techniques and Scoring System for Sleep Stages in Human Subjects. Washington, Public Health Service, US Government Printing Office, 1968.

21 Punjabi NM, Newman AB, Young TB, et al. Sleep-disordered breathing and cardiovascular disease. An outcome-based definition of hypopneas. Am J Respir Crit Care Med 2008; 177: 1150-1155.

22 Gilmartin GS, Tamisier R, Curley M, et al. Ventilatory, hemodynamic, sympathetic nervous system and vascular reactivity changes following recurrent nocturnal sustained hypoxia in humans. Am J Physiol Heart Circ Physiol 2008; 295: H778-H785.

23 Imadojemu VA, Mawji Z, Kunselman A, et al. Sympathetic chemoreflex responses in obstructive sleep apnea and effects of continuous positive airway pressure therapy. Chest 2007; 131: 1406-1413.

24 Ip MS, Tse HF, Lam B, et al. Endothelial function in obstructive sleep apnea and response to treatment. Am J Respir Crit Care Med 2004; 169: 348-353.

25 Kato M, Roberts-Thomson P, Phillips BG, et al. Impairment of endothelium-dependent vasodilation of resistance vessels in patients with obstructive sleep apnea. Circulation 2000; 102: 2607-2610.

26 Gjørup PH, Sadauskiene L, Wessels J, et al. Abnormally increased endothelin-1 in plasma during the night in obstructive sleep apnea: relation to blood pressure and severity of disease. Am J Hypertens 2007; 20: 44-52.

27 Møller DS, Lind P, Strunge B, et al. Abnormal vasoactive hormones and 24-hour blood pressure in obstructive sleep apnea. Am J Hypertens 2003; 16: 274-280.

28 Yamauchi M, Kimura H. Oxidative stress in obstructive sleep apnea: putative pathways to the cardiovascular complications. Antioxid Redox Signal 2008; 10: 755-768.

29 Dorkova Z, Petrasova D, Molcanyiova A, et al. Effects of CPAP on cardiovascular risk profile in patients with severe obstructive sleep apnea and metabolic syndrome. Chest 2008; 134: 686-692.

30 Yokoe T, Minoguchi K, Matsuo H, et al. Elevated levels of Creactive protein and interleukin-6 in patients with obstructive sleep apnea syndrome are decreased by nasal continuous positive airway pressure. Circulation 2003; 107: 1129-1134.

31 Stein PK, Pu Y. Heart rate variability, sleep and sleep disorders. Sleep Med Rev 2012; 16: 47-66.

32 Otter HP, Baust W. Experiments on cholinergic mechanisms for the control of tonic and phasic components of REM sleep. Electroenceph Clin Neurophysiol 1970; 29: 206-219.

33 Dodt C, Breckling U, Derad I, et al. Plasma epinephrine and norepinephrine concentrations of healthy humans associated with nighttime sleep and morning arousal. Hypertension 1997; 30: 71-76.

34 Kushida CA, Littner MR, Hirshkowitz M, et al. Practice parameters for the use of continuous and bilevel positive airway pressure 
devices to treat adult patients with sleep-related breathing disorders. Sleep 2006; 29: 375-380.

35 Weaver TE, Maislin G, Dinges DF, et al. Relationship between hours of CPAP use and achieving normal levels of sleepiness and daily function. Sleep 2007; 30: 711-719.

36 Barbé F, Duran-Cantolla J, Capote F, et al. Long-term effect of continuous positive airway pressure in hypertensive patients with sleep apnea. Am J Respir Crit Care Med 2010; 181: 718-726.

37 Marrone O, Salvaggio A, Bue AL, et al. Blood pressure changes after automatic and fixed CPAP in obstructive sleep apnea: relationship with nocturnal sympathetic activity. Clin Exp Hypertens 2011; 33: 373-380.

38 Narkiewicz K, van de Borne PJ, Pesek CA, et al. Selective potentiation of peripheral chemoreflex sensitivity in obstructive sleep apnea. Circulation 1999; 99: 1183-1189.
39 Sanner BM, Kollhosser P, Buechner N, et al. Influence of treatment on leptin levels in patients with obstructive sleep apnoea. Eur Respir J 2004; 23: 601-604.

40 Correia ML, Morgan DA, Sivitz WI, et al. Leptin acts in the central nervous system to produce dose-dependent changes in arterial pressure. Hypertension 2001; 37: 936-942.

41 Fietze I, Quispe-Bravo S, Hänsch T, et al. Arousal and sleep stages in patients with obstructive sleep apnoea syndrome: changes under nCPAP treatment. J Sleep Res 1997; 6: 128-133.

42 Verma A, Radtke RA, VanLandingham KE, et al. Slow wave sleep rebound and REM rebound following the first night of treatment with CPAP for sleep apnea: correlation with subjective improvement in sleep quality. Sleep Med 2001; 2: 215-223.

43 Norman RG, Pal I, Stewart C, et al. Interobserver agreement among sleep scorers from different centers in a large dataset. Sleep 2000; 23: 901-908. 